\title{
NONNEGATIVE SOLUTIONS OF TWO-POINT BOUNDARY VALUE PROBLEMS FOR NONLINEAR SECOND ORDER INTEGRODIFFERENTIAL EQUATIONS IN BANACH SPACES ${ }^{1}$
}

\author{
DAJUN GUO \\ Department of Mathematics \\ Shandong University, \\ Jinan, PEOPLES REPUBLIC OF CHINA \\ and \\ Department of Mathematics \\ University of Alberta \\ Edmonton, CANADA T6G $2 G 1$
}

\begin{abstract}
In this paper, we combine the fixed point theory, fixed point index theory and cone theory to investigate the nonnegative solutions of two-point BVP for nonlinear second order integrodifferential equations in Banach spaces. As application, we get some results for the third order case. Finally, we give several examples for both infinite and finite systems of ordinary nonlinear integrodifferential equations.
\end{abstract}

Key words: Integrodifferential equation in Banach space, measure of noncompactness, strict set contraction, fixed point index.

AMS (MOS) subject classification: $\quad 45 \mathrm{~J}, 47 \mathrm{G}$.

${ }^{1}$ Received: June, 1990. Revised: December, 1990. 


\section{Introduction}

In [1] (see also [2] Section 5.3), several existence theorems were established for the BVP of nonlinear second order differential equation in Banach space:

$$
\begin{cases}-x^{\prime \prime}=f\left(t, x, x^{\prime}\right), & 0 \leq t \leq 1 \\ a x(0)-b x^{\prime}(0)=x_{0}, & c x(1)+d x^{\prime}(1)=x_{1}\end{cases}
$$

Now, in this paper, we shall combine the fixed point theory, fixed point index theory and cone theory to extend some results of [1] to the BVP of nonlinear second order integrodifferential equation of mixed type in Banach space:

$$
\begin{cases}-x^{\prime \prime}=f\left(t, x, x^{\prime}, T x, S x\right), & 0 \leq t \leq 1 \\ a x(0)-b x^{\prime}(0)=x_{0}, & c x(1)+d x^{\prime}(1)=x_{1}\end{cases}
$$

where

$$
T x(t)=\int_{0}^{t} k(t, s) x(s) d s, \quad S x(t)=\int_{0}^{1} h(t, s) x(s) d s .
$$

We get more results about the existence of nontrivial nonnegative solutions and multiple nonnegative solutions. As application, we obtain some results for the following BVP of third order integrodifferential equation:

$$
\begin{cases}-x^{\prime \prime \prime}=f\left(t, x, x^{\prime}, x^{\prime \prime}, S x\right), & 0 \leq t \leq 1 \\ x(0)=\theta, \quad a x^{\prime}(0)-b x^{\prime \prime}(0)=x_{0}, & c x^{\prime}(1)+d x^{\prime \prime}(1)=x_{1}\end{cases}
$$

Finally, we give several examples for both infinite and finite systems of ordinary nonlinear integrodifferential equations.

\section{Several Lemmas}

Let $E$ be a real Banach space and $P$ be a cone in $E$ which defines a partial ordering in $E$ by $x \leq y$ iff $y-x \in P . P$ is said to be normal if there exists a positive constant $N$ such that $\theta \leq x \leq y$ implies $\|x\| \leq N\|y\|$, 
where $\theta$ denotes the zero element of $E$, and $N$ is called the normal constant of $P . \quad P$ is said to be solid if its interior $\operatorname{int}(P)$ is not empty. In this case, we write $x \ll y$ if $y-x \in \operatorname{int}(P)$. For details on cone theory, see [3]. In the following, $\quad I=[0,1]$.

Lemma 1. Let $P$ be a solid cone in $E, u_{0} \in E, 0 \leq t_{0} \leq t_{1} \leq 1$ and $F=\left\{x \in C[I, E]: x(t) \gg u_{0}\right.$ for $\left.t_{0} \leq t \leq t_{1}\right\}$. Then $F$ is a convex open set in $C[I, E]$.

Proof: The convexity of $F$ is obvious, so, we need only to prove that $F$ is open. Choose $v \in \operatorname{int}(P)$ and let $x_{0} \in F$. For any fixed $t^{\prime} \in\left[t_{0}, t_{1}\right]$, there exists a small $r\left(t^{\prime}\right)>0$ such that $x_{0}\left(t^{\prime}\right) \gg u_{0}+r\left(t^{\prime}\right) v$. Since $x_{0}(t)$ is continuous, there is a small interval $I\left(t^{\prime}\right)=\left(t^{\prime}-r^{\prime}, t^{\prime}+r^{\prime}\right)$ such that

$$
x_{0}(t) \gg u_{0}+r\left(t^{\prime}\right) v \text { for } t \in I\left(t^{\prime}\right) .
$$

Now, $\left\{I\left(t^{\prime}\right): t^{\prime} \in\left[t_{0}, t_{1}\right]\right\}$ forms an open covering of $\left[t_{0}, t_{1}\right]$, so, Heine-Borel theorem implies that a finite set $\left\{I\left(t_{i}\right): i=2,3, \ldots, m\right\}$ already covers $\left[t_{0}, t_{1}\right]$. Hence

$$
x_{0}(t) \gg u_{0}+r_{0} v \text { for } t \in\left[t_{0}, t_{1}\right]
$$

where $r_{0}=\min \left\{r\left(t_{2}\right), \ldots, r\left(t_{m}\right)\right\}>0$. Since $v \in \operatorname{int}(P)$, there exists $r_{1}>0$ such that

$$
v+z \geq \theta \quad \text { for any } \quad z \in E,\|z\| \leq r_{1} .
$$

Let $x \in C[I, E]$ satisfying $\left\|x-x_{0}\right\|<r_{1} r_{0}$. Then, for any $t \in\left[t_{0}, t_{1}\right]$, we have by (5) and (6),

$$
x(t)=x_{0}(t)+x(t)-x_{0}(t) \gg u_{0}+r_{0}\left[v-r_{0}^{-1}\left(x(t)-x_{0}(t)\right)\right] \geq u_{0},
$$


i.e. $x \in F$, and the proof is complete.

Corollary 1. Let $P$ be a solid cone in $E, u_{0} \in E, 0 \leq t_{0} \leq t_{1} \leq 1$ and $F=\left\{x \in C^{1}[I, E]: x(t) \gg u_{0}\right.$ for $\left.t_{0} \leq t \leq t_{1}\right\}$. Then $F$ is a convex open set in $C^{1}[I, E]$.

REMARK 1: The norm in space $C^{(m)}[I, E]$ will be defined by

$$
\|x\|_{m}=\max \left\{\|x\|_{0},\left\|x^{\prime}\right\|_{0}, \ldots,\left\|x^{(m)}\right\|_{0}\right\}
$$

where

$$
\left\|x^{(m)}\right\|_{0}=\max _{t \in I}\left\|x^{(n)}(t)\right\| \quad(n=0,1, \ldots, m)
$$

In particular, when $m=1$ we have

$$
\|x\|_{1}=\max \left\{\|x\|_{0},\left\|x^{\prime}\right\|_{0}\right\}=\max \left\{\max _{t \in I}\|x(t)\|, \max _{t \in I}\left\|x^{\prime}(t)\right\|\right\}
$$

Lemma 2. Let $H$ be a bounded set of $C^{1}[I, E]$. Suppose that $H^{\prime}=\left\{x^{\prime}\right.$ : $x \in H\}$ is equicontinuous. Then

$$
\alpha(H)=\max \left\{\sup _{t \in I} \alpha(H(t)), \sup _{t \in I} \alpha\left(H^{\prime}(t)\right)\right\}
$$

and

$$
\alpha(H)=\max \left\{\alpha(H(I)), \quad \alpha\left(H^{\prime}(I)\right)\right\}
$$

where $\alpha$ denotes the Kuratowski measure of noncompactness, $H(t)=$ $\{x(t): x \in H\}, H^{\prime}(t)=\left\{x^{\prime}(t): x \in H\right\}, H(I)=\{x(t): x \in H, t \in I\}$ and 
$H^{\prime}(I)=$

$\left\{x^{\prime}(t): x \in H, t \in I\right\}$.

Proof: (10) is known, see [2] Theorem 1.4.3. To prove (11), we first show that

$$
\alpha\left(H^{\prime}(I)\right) \leq \alpha(H)
$$

For any $\varepsilon>0, H$ can be expressed as $H=\bigcup_{i=1}^{m} S_{i}$ such that

$$
\operatorname{diam}\left(S_{i}\right)<\alpha(H)+\varepsilon, \quad i=1,2, \ldots, m
$$

Since $H^{\prime}$ is equicontinuous, there exists a partition $\left\{t_{j}\right\} \quad(j=0,1, \ldots, n)$ of $I$ such that

$$
\left\|x^{\prime}(t)-x^{\prime}(s)\right\|<\varepsilon, \quad x \in H, \quad t, s \in I_{j}=\left[t_{j-1}, t_{j}\right] \quad(j=1,2, \ldots, n) .
$$

Let $T_{i j}=\left\{x^{\prime}(t): x \in S_{i}, t \in I_{j}\right\}$, then $H^{\prime}(I)=\bigcup_{j=1}^{n} \bigcup_{i=1}^{m} T_{i j}$. For any $x^{\prime}(t)$, $y^{\prime}(s) \in T_{i j} \quad\left(x, y \in S_{i}, t, s \in I_{j}\right), \quad$ we have by (13) and (14),

$$
\begin{aligned}
\left\|x^{\prime}(t)-y^{\prime}(s)\right\| & \leq\left\|x^{\prime}(t)-x^{\prime}(s)\right\|+\left\|x^{\prime}(s)-y^{\prime}(s)\right\|<\varepsilon+\|x-y\|_{1} \\
& \leq \varepsilon+\operatorname{diam}\left(S_{i}\right)<\alpha(H)+2 \varepsilon
\end{aligned}
$$

and therefore $\operatorname{diam}\left(T_{i j}\right) \leq \alpha(H)+2 \varepsilon \quad(i=1, \ldots, m ; j=1, \ldots, n)$. Hence $\alpha\left(H^{\prime}(I)\right) \leq \alpha(H)+2 \varepsilon$, which implies (12) since $\varepsilon$ is arbitrary.

On the other hand, it is known (see [2] Lemma 1.4.4)

$$
\alpha(H(I)) \leq \alpha(H)
$$

Observing

$$
\alpha(H(t)) \leq \alpha(H(I)), \quad \alpha\left(H^{\prime}(t)\right) \leq \alpha\left(H^{\prime}(I)\right), \quad t \in I,
$$


(11) follows from (10), (12) and (15). Our lemma is proved.

REMARK 2: (a) If we only assume that $H$ is a bounded set in $C^{1}[I, E]$, then (see [2] Lemma 1.4.4)

$$
\alpha(H) \geq \alpha(H(I)), \quad \alpha(H) \geq \frac{1}{2} \alpha\left(H^{\prime}(I)\right)
$$

(b) We will also use the following conclusion (see [2] Remark 1.4.1): Let $B$ be a bounded set of $E$ and $S$ be a bounded set of real numbers. Then, for $S B=\{t x: x \in B, t \in S\}$, we have

$$
\alpha(S B)=\left(\sup _{t \in S}|t|\right) \alpha(B)
$$

(c) By the same method, we can prove the following extension of Lemma 2: Let $H$ be a bounded set of $C^{(m)}[I, E](m \geq 1)$. Suppose that $H^{(m)}=\left\{x^{(m)}: x \in H\right\}$ is equicontinuous. Then

$$
\alpha(H)=\max \left\{\sup _{t \in I} \alpha(H(t)), \sup _{t \in I} \alpha\left(H^{\prime}(t)\right), \ldots, \sup _{t \in I} \alpha\left(H^{(m)}(t)\right)\right\}
$$

and

$$
\alpha(H)=\max \left\{\alpha(H(I)), \alpha\left(H^{\prime}(I)\right), \ldots, \alpha\left(H^{(m)}(I)\right)\right\}
$$

In order to investigate BVP (2), we first consider the integral operator

$$
A x(t)=\int_{0}^{1} G(t, s) f\left(s, x(s), x^{\prime}(s), T x(s), S x(s)\right) d s+y(t)
$$

where $f \in C[I \times P \times E \times P \times P, P], y \in C^{2}[I, E], y(t) \geq \theta$ for $t \in I$ and

$$
G(t, s)= \begin{cases}J^{-1}(a t+b)(c(1-s)+d), & t \leq s \\ J^{-1}(a s+b)(c(1-t)+d), & t>s\end{cases}
$$


here $a \geq 0, b \geq 0, c \geq 0, d \geq 0$ and $J=a c+a d+b c>0$. Moreover, $T$ and $S$ are defined by (3), where $k \in C\left[D, R_{+}\right], h \in C\left[D_{0}, R_{+}\right], D=\left\{(t, s) \in R^{2}\right.$ : $a \leq s \leq t \leq 1\}$ and $D_{0}=\left\{(t, s) \in R^{2}: 0 \leq t, s \leq 1\right\}$. In the following, let $B_{R}=\{x \in P:\|x\| \leq R\}, \quad F_{R}=\{x \in E:\|x\| \leq R\}(R>0)$ and

$$
k_{0}=\max _{(t, s) \in D} k(t, s), \quad h_{0}=\max _{(t, s) \in D_{0}} h(t, s)
$$

Furthermore, let $P(I)=\left\{x \in C^{1}[I, E] \mid x(t) \geq \theta\right.$ for $\left.t \in I\right\}$. Then, $P(I)$ is a cone in $C^{1}[I, E]$. Usually, $P(I)$ is not normal even if $P$ is a normal cone in E.

Lemma 3. Let $f$ be uniformly continuous on $I \times B_{R} \times F_{R} \times B_{R} \times B_{R}$ for any $R>0$. Suppose that there exist constants $L_{i} \geq 0 \quad(i=1,2,3,4)$ with

$$
L_{1}+2 L_{2}+k_{0} L_{3}+h_{0} L_{4}<p
$$

such that

$$
\alpha(f(t, X, Y,, Z, W)) \leq L_{1} \alpha(X)+L_{2} \alpha(Y)+L_{3} \alpha(Z)+L_{4} \alpha(W)
$$

for any bonded $X, Z, W \subset P, Y \subset E$ and $t \in I$, where

$$
p=\min \left\{1, q^{-1}\right\}
$$

and

$$
q= \begin{cases}\max \left\{J^{-1}(b c+b d), J^{-1}(a d+b d), J(4 a c)^{-1}\right\}, & \text { if } a c \neq 0 \\ \max \left\{J^{-1}(b c+b d), J^{-1}(a d+b d)\right\}, & \text { if } a c=0\end{cases}
$$

Then operator $A$ (see (20)) is a strict set contraction from $P(I)$ into $P(I)$, i.e. $A$ is bounded and continuous and there exists $0<r<1$ such that $\alpha(A(Q)) \leq r \alpha(Q)$ for any bounded $Q \subset P(I)$. 
Proof: By direct differentiation of (20), we have for $x \in P(I)$,

$$
(A x(t))^{\prime}=\int_{0}^{1} G_{t}^{\prime}(t, s) f\left(s, x(s), x^{\prime}(s), T x(s), S x(s)\right) d s+y^{\prime}(t)
$$

where

$$
G_{t}^{\prime}(t, s)= \begin{cases}J^{-1} a(c(1-s)+d), & t<s \\ J^{-1}(-c)(a s+b), & t>s\end{cases}
$$

and

$$
(A x(t))^{\prime \prime}=-f\left(t, x(t), x^{\prime}(t), T x(t), S x(t)\right)+y^{\prime \prime}(t) .
$$

It is easy to see that the uniform continuity of $f$ on $I \times B_{R} \times F_{R} \times B_{R} \times B_{R}$ implies the boundedness of $f$ on $I \times B_{R} \times F_{R} \times B_{R} \times B_{R}$, so, (20) and (27) imply that $A$ is a bounded and continuous operator from $P(I)$ into $P(I)$. Now, let $Q \subset P(I)$ be bounded. By virtue of (29), $\left\{\left\|(A x(t))^{\prime \prime}\right\|: x \in Q, t \in I\right\}$ is a bounded set of $E$, so $(A(Q))^{\prime}$ is equicontinuous, and hence Lemma 2 implies (see (10))

$$
\alpha(A(Q))=\max \left\{\sup _{t \in I} \alpha(A Q(t)), \sup _{t \in I} \alpha\left((A Q)^{\prime}(t)\right)\right\}
$$

On the other hand, it is easy to know from (21), (26) and (28) that

$$
0 \leq G(t, s) \leq J^{-1}(a s+b)(c(1-s)+d) \leq q, \quad t, s \in I
$$

and

$$
\left|G_{t}^{\prime}(t, s)\right| \leq 1, \quad t, s \in I, \quad t \neq s
$$


Consequently, for $t \in I$, we have by (20), (17) and (31),

$$
\begin{aligned}
\alpha(A Q(t)) & \leq \alpha\left(\left\{\int_{0}^{1} G(t, s) f\left(s, x(s), x^{\prime}(s), T x(s), S x(s)\right) d s: x \in Q\right\}\right) \\
& \leq \alpha\left(\overline{\operatorname{co}}\left\{G(t, s) f\left(s, x(s), x^{\prime}(s), T x(s), S x(s)\right): x \in Q, s \in I\right\}\right) \\
& =\alpha\left(\left\{G(t, s) f\left(s, x(s), x^{\prime}(s), T x(s), S x(s)\right): x \in Q, s \in I\right\}\right) \\
& \leq q \alpha\left(\left\{f\left(s, x(s), x^{\prime}(s), T x(s), S x(s)\right): x \in Q, s \in I\right\}\right) \\
& \leq q \alpha\left(f\left(I, Q(I), Q^{\prime}(I), T Q(I), S Q(I)\right) .\right.
\end{aligned}
$$

Since $f$ is uniformly continuous on $I \times B_{R} \times F_{R} \times B_{R} \times B_{R}$ for any $R>0$, we have (see [2], Lemma 1.4.1)

$$
\alpha\left(f\left(I, Q(I), Q^{\prime}(I), T Q(I), S Q(I)\right)\right)=\sup _{t \in I} \alpha\left(f\left(t, Q(I), Q^{\prime}(I), T Q(I), S Q(I)\right)\right) .
$$

It follows from (33), (34) and (24) that

$$
\alpha(A Q(t)) \leq q\left\{L_{1} \alpha(Q(I))+L_{2} \alpha\left(Q^{\prime}(I)\right)+L_{3} \alpha(T Q(I))+L_{4} \alpha(S Q(I))\right\}, \quad t \in I .
$$

By (16) and (17), we have

$$
\alpha\left(Q^{\prime}(I)\right) \leq 2 \alpha(Q), \quad \alpha(Q(I)) \leq \alpha(Q)
$$

and

$$
\begin{aligned}
\alpha(T Q(I)) & =\alpha\left(\left\{\int_{0}^{t} k(t, s) x(s) d s: x \in Q, t \in I\right\}\right) \\
& \leq \alpha(\overline{\operatorname{co}}\{t k(t, s) x(s): x \in Q, s \in[0, t], t \in I\}) \\
& =\alpha(\{t k(t, s) x(s): x \in Q, s \in[0, t], t \in I\}) \\
& \leq k_{0} \alpha(Q(I)) \leq k_{0} \alpha(Q) .
\end{aligned}
$$


Similarly,

$$
\alpha(S Q(I)) \leq h_{0} \alpha(Q)
$$

Hence, (35)-(38) imply

$$
\alpha(A Q(t)) \leq q\left(L_{1}+2 L_{2}+k_{0} L_{3}+h_{0} L_{4}\right) \alpha(Q), \quad t \in I
$$

In the same way by using (27) and (32) instead of (20) and (31), we get

$$
\alpha\left((A Q)^{\prime}(t)\right) \leq\left(L_{1}+2 L_{2}+k_{0} L_{3}+h_{0} L_{4}\right) \alpha(Q), \quad t \in I
$$

It follows from (39), (40), (23) and (30) that $\alpha(A(Q)) \leq r \alpha(Q)$, where $r=\max \left\{q\left(L_{1}+2 L_{2}+k_{0} L_{3}+h_{0} L_{4}\right), L_{1}+2 L_{2}+k_{0} L_{3}+h_{0} L_{4}\right\}<1$, and our lemma is proved.

\section{Main Theorems}

Let us list some conditions for convenience

$\left(H_{1}\right) \quad x_{0} \geq \theta, x_{1} \geq \theta ; f$ is uniformly continuous on $I \times B_{R} \times F_{R} \times B_{R} \times B_{R}$ for any $\quad R>0$ and there exist $L_{i} \geq 0 \quad(i=1,2,3,4)$ such that (23) and (24) hold.

$\left(H_{2}\right) \quad \varlimsup_{R \rightarrow+\infty} \frac{M(R)}{R}<\frac{p}{m}$, where $M(R)=\sup \{\|f(t, x, y, z, w)\|:(t, x, y, z, w) \in$ $\left.I \times B_{R} \times F_{R} \times B_{R} \times B_{R}\right\}, \quad m=\max \left\{1, k_{0}, h_{0}\right\}$ and $p$ is defined by (25) and (26).

$\left(H_{3}\right)$ there exist $u_{0}>\theta$ and $0<t_{0}<t_{1}<1$ such that $f(t, x, y, z, w) \geq$ $r_{0} u_{0}$ for $t \in\left[t_{0}, t_{1}\right], x \geq u_{0}, y \in E, z \geq \theta, w \geq \theta$, where

$$
r_{0}=J\left\{\left(a t_{0}+b\right)\left(c\left(1-t_{1}\right)+d\right)\left(t_{1}-t_{0}\right)\right\}^{-1} .
$$


$\left(H_{4}\right) \quad$ cone $P$ is solid and there exist $u_{0} \gg \theta$ and $0<t_{0}<t_{1}<1$ such that $f(t, x, y, z, w) \geq r u_{0}$ for $t \in\left[t_{0}, t_{1}\right], x \geq u_{0}, y \in E, z \geq \theta, w \geq \theta$, where $r>r_{0}$ and $r_{0}$ is defined by (41).

$\left(H_{5}\right) \quad$ there exists $R_{0}>m \beta$ such that

$$
\frac{M\left(R_{0}\right)}{R_{0}}<\frac{p}{m}\left(1-\frac{m \beta}{R_{0}}\right)
$$

where

$$
\beta=J^{-1}(a+b+c+d) \max \left\{\left\|x_{0}\right\|,\left\|x_{1}\right\|\right\}
$$

Theorem 1. Let $\left(H_{1}\right)$ and $\left(H_{2}\right)$ be satisfied. Then BVP $(2)$ has at least one nonnegative solution in $C^{2}[I, E]$, i.e. solution in $C^{2}[I, E] \cap P(I)$.

Proof: It is well known, the $C^{2}[I, E]$ solution of (2) is equivalent to the $C^{1}[I, E]$ solution of the following integral equation

$$
x(t)=\int_{0}^{1} G(t, s) f\left(s, x(s), x^{\prime}(s), T x(s), S x(s)\right) d s+y(t),
$$

where $G(t, s)$ is the Green function given by $(21)$ and $y(t)$ denotes the unique solution of BVP

$$
\begin{cases}x^{\prime \prime}=\theta, & 0 \leq t \leq 1 \\ a x(0)-b x^{\prime}(0)=x_{0}, & c x(1)+d x^{\prime}(1)=x_{1}\end{cases}
$$

which is given by

$$
y(t)=J^{-1}\left\{(c(1-t)+d) x_{0}+(a t+b) x_{1}\right\} .
$$

Evidently, $\quad y \in C^{2}[I, E] \cap P(I)$. Let operator $A$ be defined by (20). Then condition $\left(H_{1}\right)$ and Lemma 3 imply that $A$ is a strict set contraction from 
$P(I)$ into $P(I)$. By $\left(H_{2}\right)$, there exist $\eta>0$ and $R_{0}>0$ such that

$$
\frac{M(R)}{R}<\frac{p}{m+\eta} \text { for } \quad R \geq m R_{0}
$$

Choose $R$ such that

$$
R>R_{0}, \quad \frac{m}{m+\eta}+\frac{\|y\|_{1}}{R}<1
$$

and let $U=\left\{x \in P(I):\|x\|_{1}<R\right\}$. For $x \in \bar{U}$, we have

$$
\|x\|_{0} \leq R, \quad\left\|x^{\prime}\right\|_{0} \leq R, \quad\|T x\|_{0} \leq k_{0} R, \quad\|S x\|_{0} \leq h_{0} R
$$

so, it follows from (20), (27), (31), (32), (47) and (48) that

$$
\begin{aligned}
\|A x\|_{0} & \leq q M(m R)+\|y\|_{0}<\frac{q p}{m+\eta} \cdot m R+\|y\|_{1} \\
& \leq\left(\frac{m}{m+\eta}+\frac{\|y\|_{1}}{R}\right) R<R
\end{aligned}
$$

and

$$
\begin{aligned}
\left\|(A x)^{\prime}\right\|_{0} & \leq M(m R)+\left\|y^{\prime}\right\|_{0}<\frac{p}{m+\eta} \cdot m R+\|y\|_{1} \\
& \leq\left(\frac{m}{m+\eta}+\frac{\|y\|_{1}}{R}\right) R<R,
\end{aligned}
$$

hence $\|A x\|_{1}<R$. Thus, we have shown

$$
A(\bar{U}) \subset U
$$

Since $\bar{U}=\left\{x \in P(1):\|x\|_{1} \leq R\right\}$ is a nonempty bounded closed convex set of $C^{1}[I, E]$, Darbo theorem (see [4]) implies that $A$ has a fixed point in $\bar{U}$, which is a solution of (44), and our theorem is proved. 
Theorem 2. Let $\left(H_{1}\right),\left(H_{2}\right)$ and $\left(H_{3}\right)$ be satisfied. Then BVP (2) has at least one nontrivial nonnegative solution $x \in C^{2}[I, E]$ which satisfies

$$
x(t) \geq u_{0}, \quad t \in\left[t_{0}, t_{1}\right] .
$$

Proof: As in the proof of Theorem 1, we can choose $R>\left\|u_{0}\right\|$ such that (51) holds. Now, let $F=\left\{x \in P(I):\|x\|_{1} \leq R\right.$ and $x(t) \geq u_{0}$ for $\left.t \in\left[t_{0}, t_{1}\right]\right\}$. Obviously, $F$ is a bounded closed convex set of $C^{1}[I, E]$, and $F$ is nonempty since $u \in F$, where $u(t) \equiv u_{0}$.

For $x \in F$, we have by $\left(H_{3}\right)$ :

$$
\begin{aligned}
t \in I & \Longrightarrow A x(t) \geq \int_{0}^{1} G(t, s) f\left(s, x(s), x^{\prime}(s), T x(s), S x(s)\right) d s \geq \theta \\
t \in\left[t_{0}, t_{1}\right] & \Longrightarrow A x(t) \geq \int_{t_{0}}^{t_{1}} G(t, s) f\left(s, x(s), x^{\prime}(s), T x(s), S x(s)\right) d s \\
& \geq\left(\int_{t_{0}}^{t_{1}} G(t, s) d s\right) r_{0} u_{0} \geq\left(\int_{t_{0}}^{t_{1}} J^{-1}\left(a t_{0}+b\right)\left(c\left(1-t_{1}\right)+d\right) d s\right) r_{0} u_{0}=u_{0}
\end{aligned}
$$

Consequently, $A x \in F$, and hence $A(F) \subset F$. Finally, again Darbo theorem implies that $A$ has a fixed point in $F$, and the proof is complete.

Theorem 3. Let cone $P$ be normal and solid. Suppose that $\left(H_{1}\right),\left(H_{2}\right),\left(H_{4}\right)$ and $\left(H_{5}\right)$ are satisfied and

$$
\left\|u_{0}\right\| \geq \frac{R_{0} N}{m}
$$

where $N$ is the normal constant of $P$. Then BVP (2) has at least three nonnegative solutions $\quad \bar{x}_{i} \in C^{2}[I, E] \quad(i=1,2,3)$ such that

$$
\left\|\bar{x}_{1}\right\|_{1}<\frac{R_{0}}{m}, \quad \bar{x}_{2}(t) \gg u_{0} \quad \text { for } \quad t \in\left[t_{0}, t_{1}\right] .
$$


Proof: By Lemma 3, $A$ is a strict set contraction from $P(I)$ into $P(I)$. It is easy to see from (46) and (43) that

$$
\|y\|_{1}=\max \left\{\|y\|_{0},\left\|y^{\prime}\right\|_{0}\right\} \leq \beta
$$

Let $U_{1}=\left\{x \in P(I):\|x\|_{1}<\frac{R_{0}}{m}\right\}$. For $x \in \bar{U}_{1}$, we have $\|x\|_{1} \leq \frac{R_{0}}{m}$. So, (42) and (56) imply

$$
\begin{aligned}
\|A x\|_{0} & \leq q M\left(R_{0}\right)+\|y\|_{0}<\frac{q p}{m}\left(R_{0}-m \beta\right)+\beta \leq \frac{1}{m}\left(R_{0}-m \beta\right)+\beta=\frac{R_{0}}{m}, \\
\left\|(A x)^{\prime}\right\|_{0} & \leq M\left(R_{0}\right)+\left\|y^{\prime}\right\|_{0}<\frac{p}{m}\left(R_{0}-m \beta\right)+\beta \leq \frac{1}{m}\left(R_{0}-m \beta\right)+\beta=\frac{R_{0}}{m} .
\end{aligned}
$$

Consequently, $\|A x\|_{1}<\frac{R_{0}}{m}$, and hence

$$
A\left(\bar{U}_{1}\right) \subset U_{1}
$$

As in the proof of Theorem 1, we can choose $R>2\left\|u_{0}\right\|$ such that (51) holds, where $U=\left\{x \in P(I):\|x\|_{1}<R\right\}$. Let $U_{2}=\left\{x \in P(I):\|x\|_{1}<R, x(t) \gg u_{0}\right.$ for $\left.t \in\left[t_{0}, t_{1}\right]\right\}$. By Corollary $1, U_{2}$ is a bounded open convex set of $P(I)$. $U_{2}$ is nonempty since $v \in U_{2}$, where $v(t) \equiv 2 u_{0}$. Moreover, we have

$$
U_{1} \subset U, \quad U_{2} \subset U, \quad U_{1} \cap U_{2}=\phi .
$$

In fact, (54) and $N \geq 1$ imply

$$
R>2\left\|u_{0}\right\| \geq \frac{2 R_{0} N}{m} \geq \frac{2 R_{0}}{m}
$$

so $U_{1} \subset U$. If $x \in U_{2}$, then $x(t) \gg u_{0}$ for $t \in\left[t_{0}, t_{1}\right]$, and therefore $N\|x\|_{1} \geq\left\|u_{0}\right\| \geq \frac{R_{0} N}{m}$, which implies $x \bar{\in} U_{2}$. So, $U_{1}$ and $U_{2}$ have no common elements, and (58) is proved. For $x \in \bar{U}_{2}$, we have $\|x\|_{1} \leq R$ and 
$x(t) \geq u_{0} \quad$ for $t \in\left[t_{0}, t_{1}\right] . \quad(51)$ implies $\|A x\|_{1}<R$, and, similar to (53), $\quad\left(H_{4}\right)$ implies:

$$
t \in\left[t_{0}, t_{1}\right] \Longrightarrow A x(t) \geq\left(\int_{t_{0}}^{t_{1}} J^{-1}\left(a t_{0}+b\right)\left(c\left(1-t_{1}\right)+d\right) d s\right) r u_{0}=\frac{r}{r_{0}} u_{0} \gg u_{0} .
$$

Hence $A x \in U_{2}$, and so

$$
A\left(\bar{U}_{2}\right) \subset U_{2}
$$

It follows from (51), (57)-(59) that the fixed point indices of strict set contraction (see [4])

$$
i(A, U, P(I))=1, \quad i\left(A, U_{k}, P(I)\right)=1 \quad(k=1,2)
$$

and

$$
\begin{aligned}
i\left(A, U \backslash\left(\bar{U}_{1} \cup \bar{U}_{2}\right), P(I)\right) & =i(A, U, P(I))-i\left(A, U_{1}, P(I)\right)-i\left(A, U_{2}, P(I)\right) \\
& =-1 .
\end{aligned}
$$

Consequently, $A$ has three fixed points $\bar{x}_{1} \in U_{1}, \bar{x}_{2} \in U_{2}, \bar{x}_{3} \in U \backslash\left(\bar{U}_{1} \cup \bar{U}_{2}\right)$, and (55) is obviously satisfied. The proof is complete.

REMARK 3: In case $x_{0}=x_{1}=\theta$, we have $\beta=0$. So, $\left(H_{5}\right)$ and (54) are satisfied if

$$
\varlimsup_{R \rightarrow+0} \frac{M(R)}{R}<\frac{p}{m}
$$

As an application, we consider the following BVP for third order integrodifferential equation:

$$
\begin{cases}-x^{\prime \prime \prime}=f\left(t, x, x^{\prime}, x^{\prime \prime}, S x\right), & 0 \leq t \leq 1 \\ x(0)=\theta, \quad a x^{\prime}(0)-b x^{\prime \prime}(0)=x_{0}, & c x^{\prime}(1)+d x^{\prime \prime}(1)=x_{1}\end{cases}
$$


Let $u=x^{\prime}$, then

$$
x(t)=x(0)+\int_{0}^{t} u(s) d s=\int_{0}^{t} u(s) d s
$$

so, (61) is reduced to the following BVP:

$$
\begin{cases}-u^{\prime \prime}=f\left(t, T_{1} u, u, u^{\prime}, S_{1} u\right), & 0 \leq t \leq 1 \\ a u(0)-b u^{\prime}(0)=x_{0}, & c u(1)+d u^{\prime}(1)=x_{1}\end{cases}
$$

where

$$
T_{1} u(t)=\int_{0}^{t} u(s) d s, \quad S_{1} u(t)=\int_{0}^{1} h_{1}(t, s) u(s) d s, \quad h_{1}(t, s)=\int_{s}^{1} h(t, r) d r .
$$

Since (62) is a BVP of type (2), we can apply above results to (61). For example, by Theorem 1 , we get the following

Theorem 4. Let $\left(H_{1}\right)$ and $\left(H_{2}\right)$ be satisfied with the change of $k_{0}=1$, $f \in C[I \times P \times P \times E \times P, P]$ and $M(R)=\sup \{\|f(t, x, y, z, w)\|:(t, x, y, z, w) \in$ $\left.I \times B_{R} \times B_{R} \times F_{R} \times B_{R}\right\}$. Then BVP (61) has at least one nonnegative solution $x \in C^{3}[I, E]$ such that $x^{\prime}(t) \geq \theta$ for $t \in I$.

\section{Examples}

This section gives several examples for both infinite and finite systems of ordinary nonlinear integrodifferential equations based on the above theorems.

EXAMPLE 1: Consider the BVP of infinite system for second order nonlinear integrodifferential equations

$$
\left\{\begin{aligned}
-x_{n}^{\prime \prime}= & \frac{16 t}{\sqrt[3]{n}}\left(x_{n}^{2}+\left(x_{n}^{\prime}\right)^{2}+3 x_{n+1} \int_{0}^{t}\left(1+e^{t-s}\right) x_{2 n}(s) d s\right)^{1 / 3} \\
& +\frac{t^{2}}{n+1}\left(t+x_{2 n-1}\right)^{1 / 3}\left(\int_{0}^{1} \sin ^{2}(t-3 s) x_{n}(s) d s\right)^{1 / 2} \ln \left(1+t\left(x_{n+1}^{\prime}\right)^{2}\right) \\
x_{n}(0)-x_{n}^{\prime}(0)= & 0, \quad x_{n}(1)+x_{n}^{\prime}(1)=0 \quad(0 \leq t \leq 1 ; n=1,2,3, \ldots) .
\end{aligned}\right.
$$


Conclusion: BVP $(63)$ has at least two nonnegative $C^{2}$ solutions $\bar{x}_{i}(t)=$ $\left(\bar{x}_{i 1}(t), \ldots, \bar{x}_{i n}(t), \ldots\right) \quad(i=1,2) \quad$ such that

$$
\bar{x}_{1 n}(t) \equiv 0 \quad(n=1,2,3, \ldots)
$$

and

$$
\begin{aligned}
& \bar{x}_{2 n}(t) \geq \frac{1}{n} \quad \text { for } \quad \frac{1}{3} \leq t \leq \frac{2}{3} \quad(n=1,2,3, \ldots) \quad \text { and } \quad \bar{x}_{2 n}(t) \rightarrow 0 \quad \text { as } \quad n \rightarrow \infty \\
& \text { for any } t \in[0,1] \text {. }
\end{aligned}
$$

Proof: We need only to prove that BVP (63) has a nonnegative solution $\bar{x}_{2}(t)$ which satisfies $(65)$ since $\bar{x}_{1}(t)=(0, \ldots, 0, \ldots)$ is evidently the trivial solution of (63). Let $E=c_{0}=\left\{x=\left(x_{1}, \ldots, x_{n}, \ldots\right): x_{n} \rightarrow 0\right\}$ with norm $\|x\|=\sup _{n}\left|x_{n}\right|$ and $P=$ $\left\{x=\left(x_{1}, \ldots, x_{n}, \ldots\right) \in c_{0}: x_{n} \geq 0, n=1,2,3, \ldots\right\}$. Then, $P$ is a normal cone of $E$ and (63) can be regarded as a BVP of the form (2), where $a=b=c=d=1$, $x_{0}=x_{1}=\theta, k(t, s)=1+e^{t-s}, h(t, s)=\sin ^{2}(t-3 s), x=\left(x_{1}, \ldots, x_{n}, \ldots\right)$, $y=\left(y_{1}, \ldots, y_{n}, \ldots\right), z=\left(z_{1}, \ldots, z_{n}, \ldots\right), w=\left(w_{1}, \ldots, w_{n}, \ldots\right)$ and $f=$ $\left(f_{1}, \ldots, f_{n}, \ldots\right)$, in which

$$
\begin{aligned}
f_{n}(t, x, y, z, w)= & \frac{16 t}{\sqrt[3]{n}}\left(x_{n}^{2}+y_{n}^{2}+3 x_{n+1} z_{2 n}\right)^{1 / 3} \\
& +\frac{t^{2}}{n+1}\left(t+x_{2 n-1}\right)^{1 / 3} w_{n}^{1 / 2} \ln \left(1+t y_{n+1}^{2}\right), \quad(0 \leq t \leq 1 ; n=1,2,3, \ldots) .
\end{aligned}
$$


By (66), we have

$$
\begin{aligned}
\left|f_{n}(t, x, y, z, w)\right| \leq & \frac{16 t}{\sqrt[3]{n}}\left(\|x\|^{2}+\|y\|^{2}+3\|x\| \cdot\|z\|\right)^{1 / 3} \\
& +\frac{t^{2}}{n+1}(t+\|x\|)^{1 / 3}\|w\|^{1 / 2} \ln \left(1+t\|y\|^{2}\right), \quad(0 \leq t \leq 1 ; n=1,2,3, \ldots),
\end{aligned}
$$

and so

$\|f(t, x, y, z, w)\| \leq 16\left(\|x\|^{2}+\|y\|^{2}+3\|x\| \cdot\|y\|\right)^{1 / 3}+\frac{1}{2}(1+\|x\|)^{1 / 3}\|w\|^{1 / 2} \ell n\left(1+\|y\|^{2}\right)$

which implies

$$
M(R) \leq 16\left(R^{2}+R^{2}+3 R^{2}\right)^{1 / 3}+\frac{1}{2}(1+R)^{1 / 3} R^{1 / 2} \ln \left(1+R^{2}\right)
$$

and consequently

$$
\lim _{R \rightarrow+\infty} \frac{M(R)}{R}=0
$$

This shows that condition $\left(H_{2}\right)$ is satisfied.

Obviously, $f \in C[I \times P \times E \times P \times P, P]$ and $f$ is uniformly continuous on $I \times B_{R} \times F_{R} \times B_{R} \times B_{R}$ for any $R>0$. We now verify that the set $f(t, X, Y, Z, W)$ is relatively compact in $E=c_{0}$ for any bounded $X, Z, W \subset$ $P, Y \subset E$ and $t \in I$. In fact, let $\left\{x^{(m)}\right\} \subset X,\left\{y^{(m)}\right\} \subset Y,\left\{z^{(m)}\right\} \subset$ $Z,\left\{w^{(m)}\right\} \subset W$ and

$$
\begin{aligned}
v_{n}^{(m)}= & f_{n}\left(t, x^{(m)}, y^{(m)}, z^{(m)}, w^{(m)}\right) . \quad \text { By }(67), \text { we have } \\
\left|v_{n}^{(m)}\right| \leq & \frac{16 t}{\sqrt[3]{n}}\left(\left\|x^{(m)}\right\|^{2}+\left\|y^{(m)}\right\|^{2}+3\left\|x^{(m)}\right\| \cdot\left\|z^{(m)}\right\|\right)^{1 / 3} \\
& +\frac{t^{2}}{n+1}\left(t+\left\|x^{(m)}\right\|\right)^{1 / 3}\left\|w^{(m)}\right\|^{1 / 2} \ln \left(1+t\left\|y^{(m)}\right\|^{2}\right), \quad(n, m=1,2,3, \ldots) .
\end{aligned}
$$

Hence $\left\{v_{n}^{(m)}\right\}$ is bounded, so, by the diagonal method, we can select a subsequence 
$\left\{m_{k}\right\}$ of $\{m\}$ such that

$$
\lim _{k \rightarrow \infty} v_{n}^{\left(m_{k}\right)}=v_{n} \quad(n=1,2,3, \ldots)
$$

It is easy to see from (70) that $v=\left(v_{1}, \ldots, v_{n}, \ldots\right) \in c_{0}$ and

$$
\left\|v^{\left(m_{k}\right)}-v\right\|=\sup _{n}\left|v_{n}^{\left(m_{k}\right)}-v_{n}\right| \rightarrow 0 \quad(k \rightarrow \infty) .
$$

Thus, $f(t, X, Y, Z, W)$ is relatively compact, and therefore $\left(H_{1}\right)$ is satisfied for $L_{1}=L_{2}=L_{3}=L_{4}=0$.

Now, let $u_{0}=\left(1, \frac{1}{2}, \ldots, \frac{1}{n}, \ldots\right)$ and $t_{0}=\frac{1}{3}, t_{1}=\frac{2}{3}$. Then $u_{0} \epsilon$ $c_{0}, u_{0}>\theta$. When $t_{0} \leq t \leq t_{1}, x \geq u_{0}, y \in c_{0}, z \geq \theta, w \geq \theta$ (i.e. $x_{n} \geq$ $\frac{1}{n}, z_{n} \geq 0, w_{n} \geq 0$ for $\left.n=1,2,3, \ldots\right)$, we have, by virtue of (66),

$$
f_{n}(t, x, y, z, w) \geq \frac{16 t}{\sqrt[3]{n}} \cdot x_{n}^{2 / 3} \geq \frac{16}{3 n}>\frac{81}{16 n} \quad(n=1,2,3, \ldots) .
$$

This shows that $\left(H_{3}\right)$ is satisfied since, by (41), $r_{0}=\frac{81}{16}$. Hence, the existence of solution $\bar{x}_{2}(t)$ satisfying (65) follows from Theorem 2 .

EXAMPLE 2: Consider the BVP of the finite system for nonlinear integrodifferential equations

$$
\left\{\begin{aligned}
-x_{n}^{\prime \prime}= & 30 t \sqrt{x}_{n} \ln \left[1+x_{n-1}+\int_{0}^{t}\left(e^{t s}+3 t^{2} s^{3}\right) x_{n+1}(s) d s\right] \\
& +\sin ^{2}\left(x_{n}^{\prime}-x_{n-1}^{\prime}+x_{n+1}\right)+\frac{1}{6}\left(x_{n}^{\prime}\right)^{2 / 3}\left(\int_{0}^{1} \cos (t-s) x_{n+1}(s) d s\right)^{1 / 3}, \\
x_{n}(0)-2 x_{n}^{\prime}(0)= & 0, \quad 3 x_{n}(1)+x_{n}^{\prime}(1)=0 \quad(0 \leq t \leq 1 ; n=1,2, \ldots, m),
\end{aligned}\right.
$$

where $x_{0}=x_{m}$ and $x_{m+1}=x_{1}$.

ConClusion: BVP $(71)$ has at least three nonnegative $C^{2}$ solutions $\bar{x}_{i}(t)=$ 
$\left(\bar{x}_{i 1}(t), \ldots, \bar{x}_{i m}(t)\right) \quad(i=1,2,3) \quad$ such that

$$
\bar{x}_{1 n}(t) \equiv 0, \quad n=1,2, \ldots, m
$$

and

$$
\bar{x}_{2 n}(t) \geq 1 \text { for } \frac{1}{4} \leq t \leq \frac{3}{4}, \quad n=1,2, \ldots, m
$$

Proof: Let $E=m$-dimensional space $E_{m}=\left\{x=\left(x_{1}, \ldots, x_{m}\right)\right\}$ with norm $\|x\|=\sup _{n}\left|x_{n}\right|$ and $P=\left\{x=\left(x_{1}, \ldots, x_{m}\right): x_{n} \geq 0, n=1,2, \ldots, m\right\}$. Then, $P$ is a normal and solid cone in $E$ and BVP (71) can be regarded as a BVP of the form (2), where $a=1, b=2, c=3, d=1, x_{0}=x_{1}=$ $\theta, k(t, s)=e^{t s}+3 t^{2} s^{3}, h(t, s)=\cos (t-s), x=\left(x_{1}, \ldots, x_{m}\right), y=\left(y_{1}, \ldots, y_{m}\right), z=$ $\left(z_{1}, \ldots, z_{m}\right), w=\left(w_{1}, \ldots, w_{m}\right)$ and $f=\left(f_{1}, \ldots, f_{m}\right)$, in which

$$
\begin{aligned}
f_{n}(t, x, y, z, w)= & 30 t \sqrt{x_{n}} \ln \left(1+x_{n-1}+z_{n+1}\right)+\sin ^{2}\left(y_{n}-y_{n-1}+x_{n+1}\right) \\
& +\frac{1}{6}\left(y_{n}\right)^{2 / 3}\left(w_{n+1}\right)^{1 / 3}, \quad n=1,2, \ldots, m .
\end{aligned}
$$

Evidently, $f \in C[I \times P \times E \times P \times P, P]$ and $\left(H_{1}\right)$ is satisfied since $f_{n}$ are continuous and $E$ is finite dimensional. We have

$$
\begin{aligned}
\left|\sin \left(y_{n}-y_{n-1}+x_{n+1}\right)\right| & \leq \min \left\{1,\left|y_{n}-y_{n-1}+x_{n+1}\right|\right\} \\
& \leq \min \left\{1,\left|y_{n}\right|+\left|y_{n-1}\right|+\left|x_{n+1}\right|\right\}
\end{aligned}
$$

so, (74) implies

$$
\begin{aligned}
\|f(t, x, y, z, w)\| \leq & 30 t \sqrt{\|x\|} \ln (1+\|x\|+\|z\|)+\min \left\{1,(2\|y\|+\|x\|)^{2}\right\} \\
& +\frac{1}{6}\|y\|^{2 / 3} \cdot\|w\|^{1 / 3}, \quad \forall t \in I, x \in P, y \in E, z \in P, w \in P
\end{aligned}
$$


and therefore

$$
M(R) \leq 30 \sqrt{R} \ln (1+2 R)+\min \left\{1,9 R^{2}\right\}+\frac{1}{6} R .
$$

Hence

$$
\varlimsup_{R \rightarrow+\infty} \frac{M(R)}{R} \leq \frac{1}{6}, \quad \varlimsup_{R \rightarrow+0} \frac{M(R)}{R} \leq \frac{1}{6}
$$

On the other hand, it is easy to see that, in this case,

$$
q=\frac{5}{6}, \quad p=1, \quad m=e+3
$$

Thus, (75) and (76) imply that $\left(H_{2}\right)$ and (60) are satisfied. Now, we check $\left(H_{4}\right)$. Let $u_{0}=(1,1, \ldots, 1)$ and $t_{0}=\frac{1}{4}, t_{1}=\frac{3}{4}$. Obviously, $u_{0} \gg \theta$ and, for $t \in\left[t_{0}, t_{1}\right]$,

$x \geq u_{0}, y \in E, z \geq \theta, w \geq \theta \quad$ (i.e. $\quad \frac{1}{4} \leq t \leq \frac{3}{4}, x_{n} \geq 1, z_{n} \geq 0, w_{n} \geq 0, n=$ $1,2, \ldots, m), \quad(74)$ implies

$$
f_{n}(t, x, y, z, w) \geq 30 t \sqrt{x_{n}} \ln \left(1+x_{n-1}\right) \geq \frac{15}{2} \ln 2 \quad(n=1,2, \ldots, m) .
$$

On the other hand, we have by (41)

$$
r_{0}=\frac{320}{63}<\frac{15}{2} \ln 2
$$

Hence, (77) and (78) imply that $\left(H_{4}\right)$ is satisfied with $r=\frac{15}{2} \ell n 2$. Finally, our conclusion follows from Theorem 3 and Remark 3.

EXAMPLE 3: Consider the BVP of infinite system for third order nonlinear inte- 
grodifferential equation

$$
\left\{\begin{aligned}
&-x_{n}^{\prime \prime \prime}= \frac{t^{2}}{n(n+1)} x_{n+1}^{1 / 3}\left(1-2 t-x_{n}^{\prime}\right)^{2 / 3} \\
&+\frac{1}{(t+1) \sqrt{n}}\left[\left(x_{2 n}+\sin t\right)^{3}+\left(x_{n+1}^{\prime \prime}\right)^{2} \int_{0}^{1} e^{-t s} \cos (t-s) x_{n}(s) d s\right]^{1 / 4}, \\
& x_{n}(0)=0, \quad 2 x_{n}^{\prime}(0)-x_{n}^{\prime \prime}(0)=\frac{1}{n}, \quad x_{n}^{\prime}(1)+2 x_{n}^{\prime \prime}(1)=\frac{1}{n^{2}} \quad(0 \leq t \leq 1 ; n=1,2,3, \ldots) .
\end{aligned}\right.
$$

Conclusion: BVP (79) has at least one nonnegative $C^{3}$ solution $\bar{x}(t)=\left(\bar{x}_{1}(t), \ldots, \bar{x}_{n}(t)\right.$, such that $x_{n}^{\prime}(t) \geq 0(n=1,2,3, \ldots)$ and $\bar{x}_{n}(t) \rightarrow 0$ as $n \rightarrow \infty$ for any $t \in[0,1]$.

Proof: Let $E=c_{0}$ and $P=\left\{x=\left(x_{1}, \ldots, x_{n}, \ldots\right) \in c_{0}: x_{n} \geq 0, n=\right.$ $1,2,3, \ldots\}$ as in Example 1. Then, (79) can be regarded as a BVP of the form (61), where

$a=d=2, \quad b=c=1, \quad x_{0}=\left(1, \frac{1}{2}, \ldots, \frac{1}{n}, \ldots\right) \in c_{0}, \quad x_{1}=\left(1, \frac{1}{2^{2}}, \ldots, \frac{1}{n^{2}}, \ldots\right) \in$

$c_{0}$

$h(t, s)=e^{-t s} \cos (t-s), x=\left(x_{1}, \ldots, x_{n}, \ldots\right), y=\left(y_{1}, \ldots, y_{n}, \ldots\right), z=\left(z_{1}, \ldots, z_{n}, \ldots\right)$,

$w=\left(w_{1}, \ldots, w_{n}, \ldots\right), f=\left(f_{1}, \ldots, f_{n}, \ldots\right)$, in which

$$
\begin{aligned}
f_{n}(t, x, y, z, w)= & \frac{t^{2}}{n(n+1)} x_{n+1}^{1 / 3}\left(1-2 t-y_{n}\right)^{2 / 3} \\
& +\frac{1}{(t+1) \sqrt{n}}\left[\left(x_{2 n}+\sin t\right)^{3}+z_{n+1}^{2} w_{n}\right]^{1 / 4}, \quad(n=1,2,3, \ldots) .
\end{aligned}
$$

Now, (80) implies

$$
\|f(t, x, y, z, w)\| \leq \frac{1}{2}\|x\|^{1 / 3}(1+\|y\|)^{2 / 3}+\left[(\|x\|+1)^{3}+\|z\|^{2} \cdot\|w\|\right]^{1 / 4}
$$

so

$$
M(R) \leq \frac{1}{2} R^{1 / 3}(1+R)^{2 / 3}+\left[(R+1)^{3}+R^{3}\right]^{1 / 4}
$$


and therefore

$$
\varlimsup_{R \rightarrow+\infty} \frac{M(R)}{R} \leq \frac{1}{2}
$$

This shows that $\left(H_{2}\right)$ is satisfied since it is easy to calculate that $p=m=1$.

On the other hand, similar to Example 1, we can check that $f(t, X, Y, Z, W)$ is relatively compact in $E=c_{0}$ for any bounded $X, Y, W \subset P, Z \subset E$ and $t \in I$, so $\left(H_{1}\right)$ is also satisfied.

Finally, our conclusion follows from Theorem 4.

\section{REFERENCES}

1. J. Chandra, V. Lakshmikantham and A.R. Mitchell, Existence of solutions of boundary value problems for nonlinear second order systems in a Banach space, Nonlinear Anal. 2 (1978), 157-168.

2. V. Lakshmikantham and S. Leela, "Nonlinear Differential Equations in Abstract Spaces," Pergamon Press, Oxford, 1981.

3. D. Guo and V. Lakshmikantham, "Nonlinear Problems in Abstract Cones," Academic Press Boston, New York, 1988.

4. D. Guo, "Nonlinear Functional Analysis," Shandong Sci. and Tech. Press, Jinan, 1985 (in Chinese). 


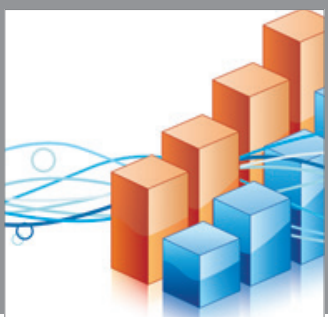

Advances in

Operations Research

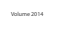

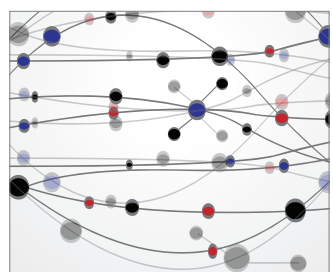

\section{The Scientific} World Journal
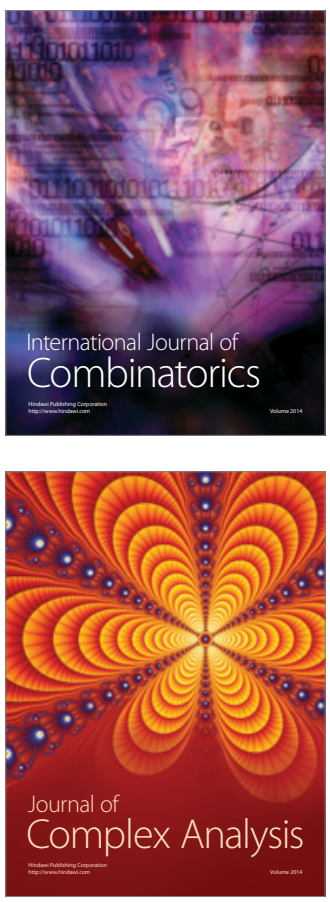

International Journal of

Mathematics and

Mathematical

Sciences
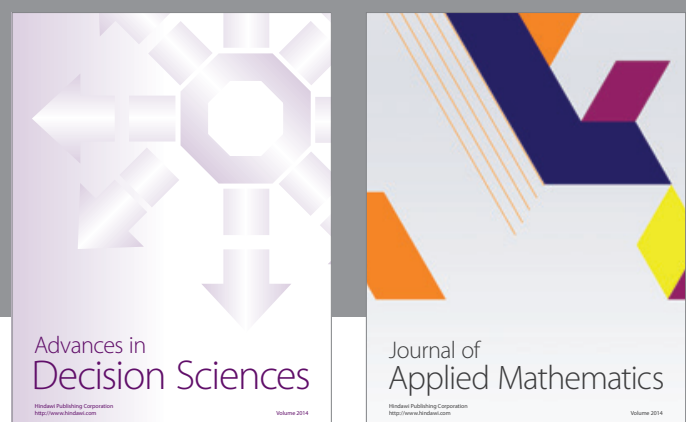

Journal of

Applied Mathematics
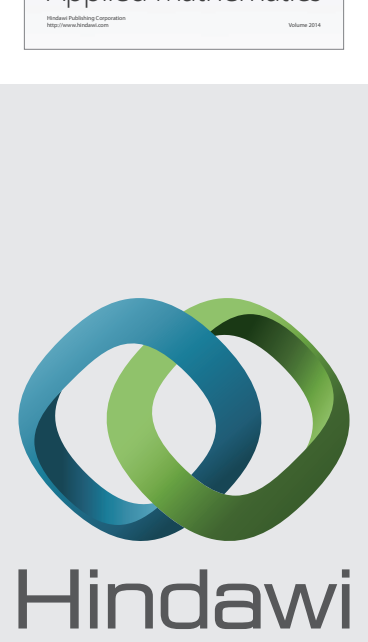

Submit your manuscripts at http://www.hindawi.com
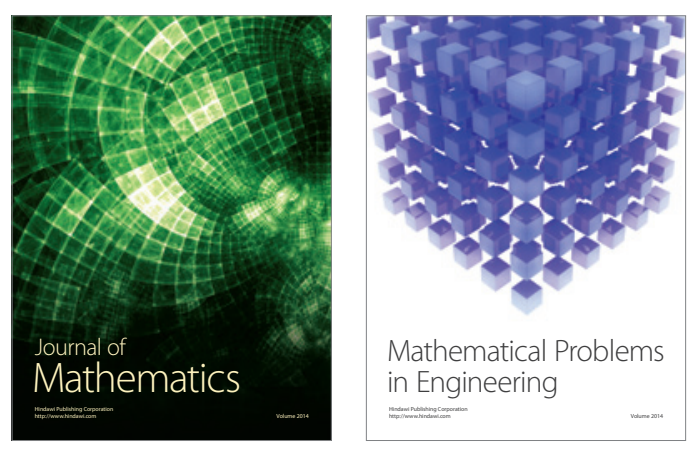

Mathematical Problems in Engineering
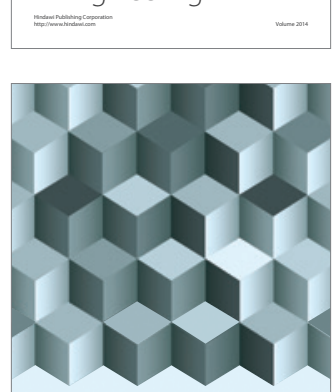

Journal of

Function Spaces
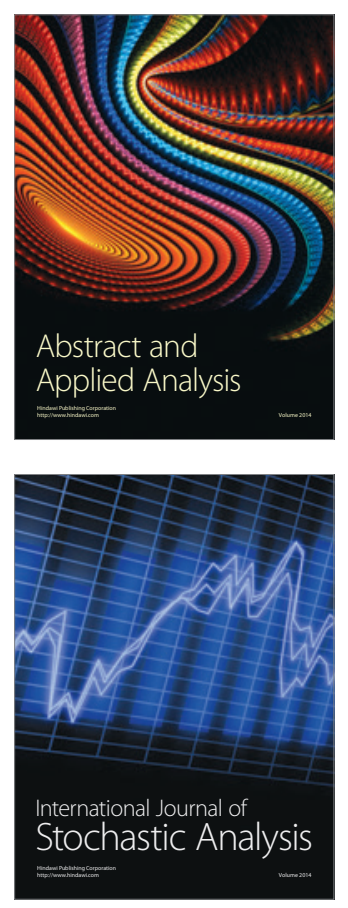

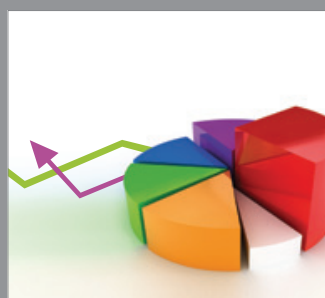

ournal of

Probability and Statistics

Promensencen
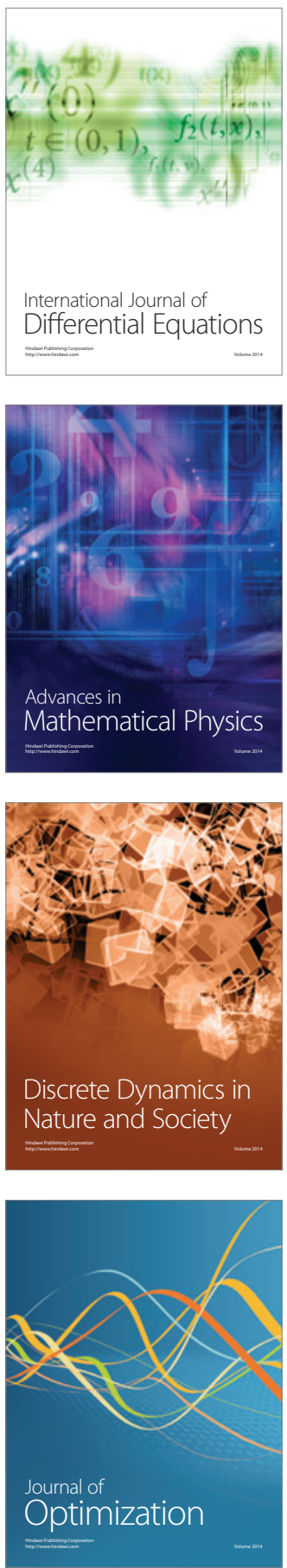